\title{
The Dramatic Recovery of a Patient with Biguanide- associated Severe Lactic Acidosis Following Thiamine Supplementation
}

\author{
Shigeo Godo ${ }^{1,2}$, Yoshitaro Yoshida ${ }^{2}$, Motoo Fujita ${ }^{2}$, Daisuke Kudo ${ }^{2,3}$, \\ Ryosuke Nomura $^{2}$, Hiroaki Shimokawa ${ }^{1}$ and Shigeki Kushimoto ${ }^{2,3}$
}

\begin{abstract}
Biguanides are a drug of choice for the treatment of type 2 diabetes mellitus. Although they can cause lactic acidosis in susceptible patients with predisposing risk factors, the incidence of lactic acidosis is reported to be very low when they are used properly. We herein present a case of biguanide-associated severe lactic acidosis complicated with thiamine deficiency that was provoked without predisposing factors for thiamine deficiency. Diabetic patients taking biguanide may be predisposed to thiamine deficiency, even when there is no evidence of risk factors, and the high-dose administration of thiamine may be essential in the treatment of this otherwise under-recognized disorder.
\end{abstract}

Key words: buformin, biguanide, lactic acidosis, thiamine deficiency

(Intern Med 56: 455-459, 2017)

(DOI: 10.2169/internalmedicine.56.7754)

\section{Introduction}

Unless contraindicated, biguanides are recommended as a first-line therapy for the treatment of type 2 diabetes mellitus (1). They exert their antihyperglycemic effects, in part, by decreasing hepatic gluconeogenesis from lactate and pyruvate, while they can increase lactate production through the inhibition of mitochondrial respiration and by promoting the conversion of glucose to lactate, which leads to lactic acidosis (2). The contraindications for the use of biguanides include severe renal or hepatic insufficiency, extreme old age, and serious acute illnesses involving circulatory dysfunction, because they are associated with a high risk of lactic acidosis (2). However, the incidence of biguanideassociated lactic acidosis has been reported to be very low when biguanides are properly used (3). Thiamine deficiency is important in the differential diagnosis of lactic acidosis, and has been recognized as a distinct clinical entity from biguanide-associated lactic acidosis (4). We herein present the case of a patient with biguanide-associated severe lactic acidosis who showed a dramatic improvement after the high-dose administration of thiamine. When diabetic patients taking biguanide present with lactic acidosis, thiamine deficiency should be suspected as a comorbidity and high-dose thiamine should be administered without delay as a diagnostic treatment.

\section{Case Report}

A 75-year-old woman was referred to our department due to sudden-onset shock of unknown origin with severe lactic acidosis. One year prior to her presentation, the patient experienced a cerebral infarction, which resulted in lefthemiparesis and mild cognitive impairment (Mini-Mental State Examination score: 24/30); she also had diabetes mellitus, which was treated with buformin $(100 \mathrm{mg} /$ day $)$ and sitagliptin $(50 \mathrm{mg} / \mathrm{day})$. She was also prescribed cilostazol $(100 \mathrm{mg} / \mathrm{day})$ for the secondary prevention of cerebral infarction, and lansoprazole (30 mg/day) and magnesium ox-

\footnotetext{
${ }^{1}$ Department of Cardiovascular Medicine, Tohoku University Graduate School of Medicine, Japan, ${ }^{2}$ Department of Emergency and Critical Care Medicine, Tohoku University Hospital, Japan and ${ }^{3}$ Division of Emergency and Critical Care Medicine, Tohoku University Graduate School of Medicine, Japan

Received for publication May 24, 2016; Accepted for publication June 10, 2016

Correspondence to Dr. Shigeo Godo, s-godo@cardio.med.tohoku.ac.jp
} 
ide (660 mg/day). Following the stroke event she had lived in a geriatric facility and had consumed a regular diet. She had remained in her usual state of health, and had not shown any significant weight changes, reduced dietary intake or specific gastrointestinal symptoms. She did not drink alcohol and her kidney and liver functions had been normal. Three hours before her presentation, she complained of dyspnea at rest and was transferred to another hospital, where she arrived with severe hypotension (blood pressure $70 / 36 \mathrm{mmHg}$, pulse 135 beats/min) with profound lactic acidosis ( $\mathrm{pH} 7.108$, base excess $-24.6 \mathrm{mmol} / \mathrm{L}$, lactate 18 $\mathrm{mmol} / \mathrm{L}$ ). On presentation to our department, a physical examination revealed the following, blood pressure, 60/45 $\mathrm{mmHg}$; pulse, 158 beats/min; respiratory rate, 30 breaths/ min; body temperature, $34.8^{\circ} \mathrm{C}$, and oxygen saturation, $98 \%$ with $5 \mathrm{~L} / \mathrm{min}$ oxygen delivered through a face mask. Her height, weight and body mass index were $145 \mathrm{~cm}, 55.2 \mathrm{~kg}$, and $26.3 \mathrm{~kg} / \mathrm{m}^{2}$, respectively. Although she presented with a decreased level of consciousness (Glasgow Coma Scale score of E2V4M4), she did not show specific symptoms of polyneuropathy or Wernicke's encephalopathy. She required immediate mechanical ventilation under endotracheal intubation due to the worsening of her mental state and hemodynamic instability. Echocardiography revealed biventricular systolic dysfunction with a left ventricular ejection fraction that was as low as $30 \%$. Acute coronary syndrome and myocarditis were excluded because emergency coronary angiography revealed no significantly diseased vessels and an endomyocardial biopsy specimen from the right ventricle revealed no evidence of inflammatory infiltration. The patient's hemodynamic parameters were as follows: mean pulmonary capillary wedge pressure, $18 \mathrm{mmHg}$; pulmonary artery pressure, $36 / 25 \mathrm{mmHg}$ (mean, $26 \mathrm{mmHg}$ ); right atrial pressure, 30/24 $\mathrm{mmHg}$; cardiac output, $3.0 \mathrm{~L} / \mathrm{min}$; cardiac index, $2.0 \mathrm{~L} / \mathrm{min} / \mathrm{m}^{2}$; systemic vascular resistance, 1,047 dyn $\cdot \mathrm{sec} / \mathrm{cm}^{5}$; and pulmonary vascular resistance, $215 \mathrm{dyn} \cdot \mathrm{sec} / \mathrm{cm}$ ${ }^{5}$. Based on her medical history of buformin therapy and the preliminary clinical data, our initial diagnosis was severe lactic acidosis resulting from the toxic effects of buformin and cardiogenic shock due to cardiomyopathy of unknown etiology. We initiated the conventional treatment for biguanide-associated lactic acidosis with continuous renal replacement therapy and the continuous infusion of $8.4 \%$ sodium bicarbonate. Because echocardiography and cardiac catheterization examinations showed marked biventricular systolic dysfunction and a low cardiac output with relatively low systemic vascular resistance, we initiated multiple inotropic and vasopressor support, including dobutamine (10 $\mu \mathrm{g} / \mathrm{kg} / \mathrm{min})$, dopamine $(3 \mu \mathrm{g} / \mathrm{kg} / \mathrm{min})$, noradrenaline $(0.3 \mu \mathrm{g} /$ $\mathrm{kg} / \mathrm{min}$ ), and further added vasopressin (2 units/h), hydrocortisone (200 mg/day) and an intra-aortic balloon pump in order to maintain a mean atrial pressure of at least 65 $\mathrm{mmHg}$. Although her hypotension and acidemia gradually improved, the profound hyperlactatemia persisted without any significant improvement (Figure). On the second morning (17 hours after her arrival), because we had not obtained definite evidence of anaerobic metabolic disorders that would have caused persistent severe hyperlactatemia or a good clinical response to the conventional therapies for biguanide toxicity, we suspected thiamine deficiency as an alternative cause of her persistent severe lactic acidosis and administered high-dose fursultiamine $(100 \mathrm{mg})$, a thiamine derivative, intravenously. The response to the thiamine replacement therapy was drastic; the patient's blood pressure rapidly increased, the reduced left ventricular ejection fraction was gradually restored to the normal range, the hyperlactatemia fully recovered within 24 hours, and all of the hemodynamic and renal support could be tapered off within a few days (Figure). We continued to administer fursultiamine (300 mg/day, parenterally) for five days, after which we switched to oral supplementation. On day 9, she was successfully extubated, and had recovered to her premorbid state; her cardiac functions normalized as well. The serum concentration of vitamin B1, which was analyzed from a blood sample that had been obtained just before the first administration of fursultiamine, was $23 \mathrm{ng} / \mathrm{mL}$ (reference range: $26-58 \mathrm{ng} / \mathrm{mL}$ ) (Table). The final diagnosis was thiamine deficiency syndrome due to the toxic effects of buformin.

\section{Discussion}

The present case highlighted two important clinical issues. Diabetic patients taking biguanide are predisposed to thiamine deficiency, even if they have no disease-related factors that might provoke thiamine deficiency, and the intravenous infusion of high-dose thiamine may be essential for the treatment of this disorder. To the best of our knowledge, this is the first case report to demonstrate biguanide-associated lactic acidosis complicated with thiamine deficiency in a diabetic patient without predisposing risk factors for thiamine deficiency.

First, diabetic patients taking biguanide may be at risk of developing thiamine deficiency syndrome. Although our patient was in the old-old age range, she had no predisposing factors for biguanide-associated lactic acidosis (these include chronic renal insufficiency, congestive heart failure, alcohol abuse and overdose) (2). That biguanides can cause lactic acidosis, especially in very elderly patients with advanced chronic kidney disease is well known in clinical practice (5). However, the need for caution with regard to thiamine deficiency when diabetic patients without predisposing factors for thiamine deficiency take biguanide has never been emphasized. Given that biguanides have been widely used as a leading anti-hyperglycemic agent in the current era (1), this caution provides important clinical implications. Although it is inconclusive, the lactic acidosis in our patient may be attributable to thiamine deficiency, the toxic effects of biguanide or both. In Japan, buformin is contraindicated for patients who are $\geq 75$ years of age due to the increased risk of lactic acidosis. This raises the possibility that the off-label use of buformin, at least in part, contributed to the develop- 


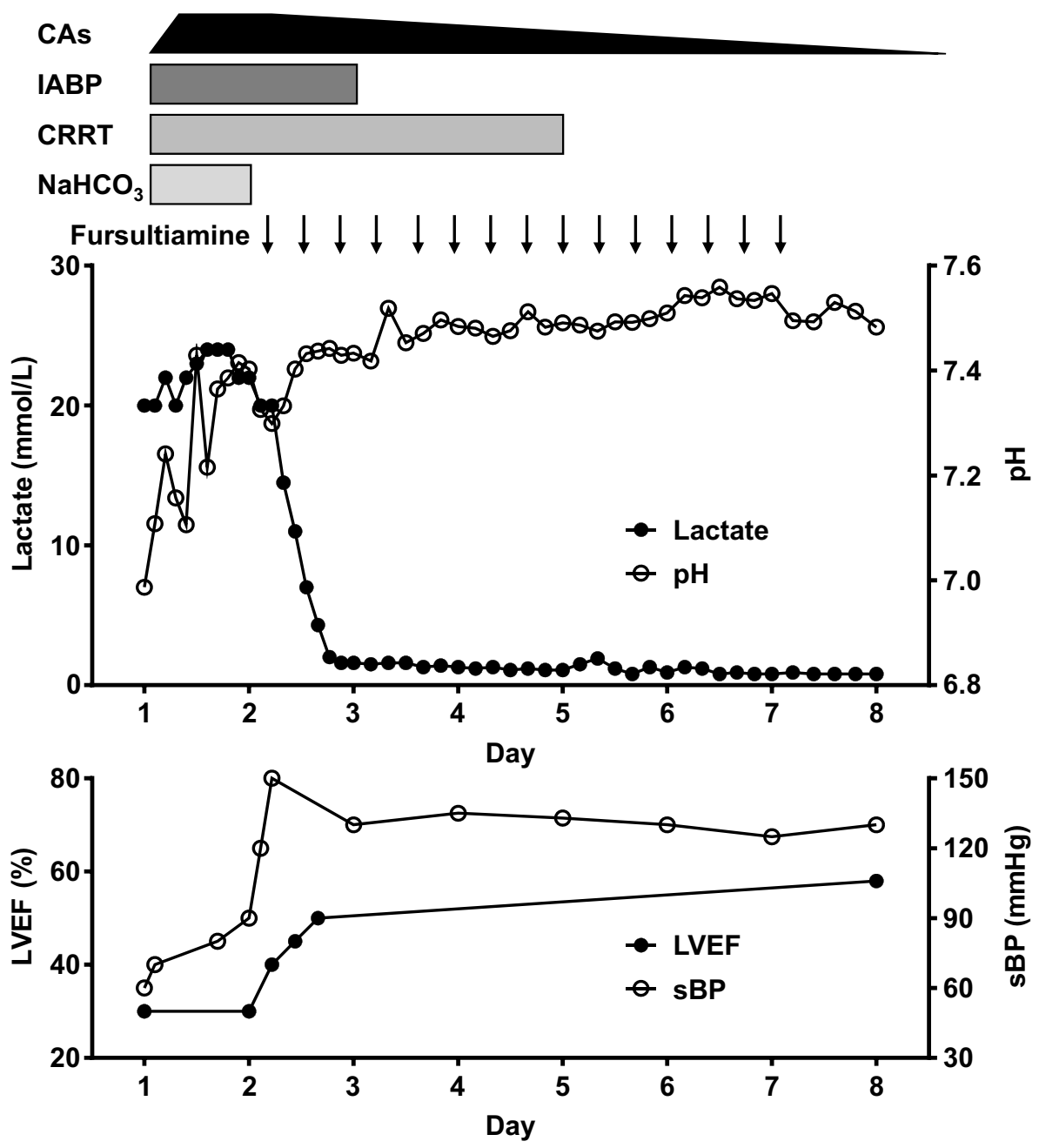

Figure. The clinical course of the patient's hemodynamic status, circulatory management, and lactic acidosis. The patient's marked lactic acidosis and the reduction in her left ventricular systolic function were dramatically reversed by the administration of high-dose thiamine. Each arrow indicates the intravenous administration of fursultiamine $(100 \mathrm{mg})$, a thiamine derivative. CAs: denotes catecholamines, CRRT: continuous renal replacement therapy, IABP: intra-aortic balloon pump, LVEF: left ventricular ejection fraction, $\mathrm{NaHCO}_{3}$ : sodium bicarbonate, sBP: systolic blood pressure

ment of lactic acidosis through the toxic effects of biguanide in our patient. However, the drastic response to the administration of thiamine suggests that the thiamine deficiency could have been involved in the pathophysiology of severe lactic acidosis. The further accumulation of cases is needed to elucidate whether biguanides can directly cause thiamine deficiency in diabetic patients without predisposing factors for this condition.

Second, the intravenous infusion of high-dose thiamine is useful for the treatment and shows a high diagnostic value in the management of patients with this disorder. The patient's hyperlactatemia, which was refractory to the conventional treatment for lactic acidosis, including renal replacement therapy, promptly responded to administration of thiamine. Cardiac beriberi is a fulminant form of thiamine deficiency syndrome that is associated with ventricular systolic dysfunction and marked vasodilatation, which leads to hemodynamic collapse (6). In rare cases, this cardiac dysfunction manifests as low cardiac output, such as was ob- served in our patient. However, the treatment is the same: high-dose thiamine $(7,8)$. In most cases, thiamine deficiency occurs in patients with preexisting comorbidities and metabolic stresses (9) such as alcohol abuse $(7,10)$, dialysis, infection (11), the long-term use of diuretics and malnutrition (12). These predisposing factors for thiamine deficiency serve as an important diagnostic clue in the clinical setting and - in all cases - suspected thiamine deficiency can be immediately treated with high-dose thiamine without any significant adverse effects. The level of vitamin B1 in patients with thiamine deficiency is not necessarily extremely low. In the present case, the patient's serum concentration of vitamin B1 was only marginally lower than the normal range, which is consistent with previous reports of thiamine deficiency syndrome $(7,10,11)$. However, because our patient did not present any predisposing factors for thiamine deficiency, we initially considered that her lactic acidosis was attributable to the toxic effects of buformin rather than thiamine deficiency. This resulted in a one-day delay in the ad- 
Table. Laboratory Data.

\begin{tabular}{|c|c|c|c|}
\hline & Reference range & On admission & On discharge \\
\hline \multicolumn{4}{|l|}{ Urinalysis } \\
\hline Glucose & & - & \pm \\
\hline Ketone body & & $1+$ & - \\
\hline \multicolumn{4}{|l|}{ Arterial blood gas analysis } \\
\hline Fraction of inspired oxygen & & 0.60 & 0.21 \\
\hline $\mathrm{pH}$ & & 6.987 & 7.483 \\
\hline $\mathrm{pO}_{2}(\mathrm{mmHg})$ & & 79.0 & 104 \\
\hline $\mathrm{pCO}_{2}(\mathrm{mmHg})$ & & 24.4 & 37.9 \\
\hline $\mathrm{HCO}_{3}{ }^{-}(\mathrm{mmol} / \mathrm{L})$ & & 5.6 & 28.1 \\
\hline Base excess $(\mathrm{mmol} / \mathrm{L})$ & & -24.9 & 4.8 \\
\hline Anion gap (mmol/L) & & 26.8 & 10.2 \\
\hline \multicolumn{4}{|l|}{ Others } \\
\hline Lactate $(\mathrm{mmol} / \mathrm{L})$ & $0.37-1.65$ & 20.0 & 0.8 \\
\hline BUN (mg/dL) & $8.0-20.0$ & 46.0 & 14.0 \\
\hline Cre $(\mathrm{mg} / \mathrm{dL})$ & $0.32-0.84$ & 1.10 & 0.47 \\
\hline $\mathrm{eGFR}\left(\mathrm{mL} / \mathrm{min} / 1.73 \mathrm{~m}^{2}\right)$ & $>60$ & 37 & 95 \\
\hline $\mathrm{BNP}(\mathrm{pg} / \mathrm{mL})$ & $<18.4$ & 104 & 249 \\
\hline Plasma glucose (mg/dL) & $68.0-109$ & 220 & 155 \\
\hline Vitamin B1 (ng/mL) & $26.0-58.0$ & 23.0 & 101 \\
\hline Vitamin B2 (ng/mL) & $128-276$ & 196 & NA \\
\hline Vitamin B12 (pg/mL) & $180-914$ & 327 & NA \\
\hline Folic acid (ng/mL) & $>4.0$ & 5.3 & NA \\
\hline
\end{tabular}

ministration of thiamine. The role of thiamine in critically ill patients has attracted much attention as a 'metabolic resuscitator'. This was well exemplified in a randomized trial of high-dose thiamine for patients with septic shock and hyperlactatemia, which showed that thiamine treatment may be beneficial in a subgroup of patients with baseline thiamine deficiency (13). Notably, more than one third of the study cohort exhibited absolute thiamine deficiency at baseline (13). In addition, the current guidelines on parenteral nutrition recommend that $100-300 \mathrm{mg} /$ day of thiamine be supplemented intravenously during the initial 3 days of treatment in critically ill patients with possible thiamine deficiency (14). Thiamine supplementation is a safe, easily available and well-tolerated therapy with few adverse effects other than anaphylaxis (11).

We suggest two possible mechanisms by which buformin could have played a role in the development of thiamine deficiency in our patient. First, biguanides may have an immediate anti-thiamine effect (15). This notion is based on data from animal studies, which have shown that phenformin induces hyperlactatemia through the inhibitory effects on thiamin activity (15). Second, biguanides may interfere with thiamine absorption. More recently, it has been reported that when administered at their therapeutic doses, metformin and phenformin inhibit hTHTR-2, a human thiamine transporter, suggesting that biguanides may cause clinically relevant thiamine deficiency by reducing the absorption of thiamine (16). Moreover, it has been reported that diabetic patients frequently show decreased plasma concentrations of thiamine due to the enhanced urinary excretion of thiamine (17). It is well-documented that metformin causes vitamin B12 deficiency over several years (18-20). The precise mechanisms by which biguanides interfere with thiamine to cause lactic acidosis warrant further examination.

Thiamine deficiency remains an under-recognized condition and a diagnostic pitfall with major clinical implications (21). The course of our patient provided two important clinical suggestions; diabetic patients taking biguanide are predisposed to thiamine deficiency, even if they have no factors to provoke the deficiency, and that the rapid administration of high-dose thiamine is essential for the treatment and diagnosis of this disorder, which is otherwise prone to be medical oversight. In conclusion, critical care clinicians should consider the possibility of thiamine deficiency in patients with biguanide-associated lactic acidosis, and administer high-dose thiamine without delay as a diagnostic treatment.

The authors state that they have no Conflict of Interest (COI).

\section{References}

1. Chamberlain JJ, Rhinehart AS, Shaefer CF Jr, Neuman A. Diagnosis and Management of Diabetes: Synopsis of the 2016 American Diabetes Association Standards of Medical Care in Diabetes. Ann Intern Med 164: 542-552, 2016.

2. DeFronzo R, Fleming GA, Chen K, Bicsak TA. Metforminassociated lactic acidosis: current perspectives on causes and risk. Metabolism 65: 20-29, 2016.

3. Misbin RI. The phantom of lactic acidosis due to metformin in patients with diabetes. Diabetes Care 27: 1791-1793, 2004.

4. Kraut JA, Madias NE. Lactic acidosis. N Engl J Med 371: 23092319, 2014.

5. Inzucchi SE, Lipska KJ, Mayo H, Bailey CJ, McGuire DK. Metformin in patients with type 2 diabetes and kidney disease: a systematic review. JAMA 312: 2668-2675, 2014.

6. Attas M, Hanley HG, Stultz D, Jones MR, McAllister RG. Fulminant beriberi heart disease with lactic acidosis: presentation of a 
case with evaluation of left ventricular function and review of pathophysiologic mechanisms. Circulation 58: 566-572, 1978.

7. Imamura T, Kinugawa K. Shoshin beriberi with low cardiac output and hemodynamic deterioration treated dramatically by thiamine administration. Int Heart J 56: 568-570, 2015.

8. McIntyre N, Stanley NN. Cardiac beriberi: two modes of presentation. Br Med J 3: 567-569, 1971.

9. Luft FC. Lactic acidosis update for critical care clinicians. J Am Soc Nephrol 12: S15-S19, 2001.

10. Yamaguchi H, Iima T, Shirakami A, et al. A case of type 2 diabetes mellitus with severe lactic acidosis and beriberi heart disease during the administration of low-dose metformin. Tonyobyo (Journal of the Japan Diabetes Society) 57: 188-196, 2014 (in Japanese, Abstract in English).

11. Sechi G, Serra A. Wernicke's encephalopathy: new clinical settings and recent advances in diagnosis and management. Lancet Neurol 6: 442-455, 2007

12. Kawano H, Koide Y, Toda G, Yano K. ST-segment elevation of electrocardiogram in a patient with Shoshin beriberi. Intern Med 44: 578-585, 2005.

13. Donnino MW, Andersen LW, Chase M, et al. Randomized, double-blind, placebo-controlled trial of thiamine as a metabolic resuscitator in septic shock: a pilot study. Crit Care Med 44: 360367, 2016.

14. Singer P, Berger MM, Van den Berghe G, et al. ESPEN Guide- lines on Parenteral Nutrition: intensive care. Clin Nutr 28: 387400, 2009.

15. Alston TA. Does metformin interfere with thiamine? Arch Intern Med 163: 983, 2003.

16. Liang X, Chien HC, Yee SW, et al. Metformin is a substrate and inhibitor of the human thiamine transporter, THTR-2 (SLC19A3). Mol Pharm 12: 4301-4310, 2015.

17. Thornalley PJ, Babaei-Jadidi R, Al Ali H, et al. High prevalence of low plasma thiamine concentration in diabetes linked to a marker of vascular disease. Diabetologia 50: 2164-2170, 2007.

18. Gilligan MA. Metformin and vitamin B 12 deficiency. Arch Intern Med 162: 484-485, 2002.

19. Andres E, Noel E, Goichot B. Metformin-associated vitamin B12 deficiency. Arch Intern Med 162: 2251-2252, 2002.

20. Tomkin GH, Hadden DR, Weaver JA, Montgomery DA. VitaminB12 status of patients on long-term metformin therapy. Br Med J 2: 685-687, 1971.

21. Amrein K, Ribitsch W, Otto R, Worm HC, Stauber RE. Severe lactic acidosis reversed by thiamine within 24 hours. Crit Care 15: 457, 2011.

The Internal Medicine is an Open Access article distributed under the Creative Commons Attribution-NonCommercial-NoDerivatives 4.0 International License. To view the details of this license, please visit (https://creativecommons.org/licenses/ by-nc-nd/4.0/).

(C) 2017 The Japanese Society of Internal Medicine http://www.naika.or.jp/imonline/index.html 